Supplement Issue 1: International Tehran Breast Cancer Congress (TBCC9)

\title{
Examining the Expression of the Protein in GPX-1 Breast Cancer Tissue Cells
}

\section{Saeed Sorush ${ }^{1, *}$, Mehrnaz Ajorloo ${ }^{2}$}

${ }^{1}$ Gilan University of Medical Sciences, Rasht, Iran

${ }^{2}$ Rudehen Azad University, Rudehen, Iran

* Corresponding author: Saeed Sorush, Gilan University of Medical Sciences, Rasht,

DOI: $10.21859 / \mathrm{mci}-$ supp-21 Iran.E-mail: saeed.sorush55@yahoo.com

\section{Keywords:}

GPX-1

Breast Cancer

Tissue Cells

\begin{abstract}
Introduction: The most common type of cancer among women is breast cancer, which is always a serious threat to all ages. In general, factors such as the age of the person,the birth of the first child, the use of contraceptives, people with low activity, inactive people and overweights are involved in the process of breast cancer. According to statistics, only 5 to $10 \%$ of cases of breast cancer are affected by the transmission of genetic factors. This GPX-1 protein is produced by people of the same genome, including gene proteins which act as an enzyme for glutathione peroxidase and is effective in eliminating intracellular free radicals. As you know, glutathione peroxidase of a selenoprotein has a direct relationship with breast cancer, which is associated with an increase in the amount of selenium enzyme activity, the risk factors for breast cancer also increase. This is due to the displacement of amino acids 5 alanine and the replacement of amino acids 6 and 7 of alanine in the position of 198 proteins of leucine.

Conclusions: While changing the position of selenocysteine in the active site of the enzyme, we always we see a decrease in the activity of the enzyme, which explains the association between the expression of this protein and the development of cancer. The change in the position of selenocysteine in the active site of SKBR3 in the GPX-1 cells of the enzyme and subsequent reduction of the enzyme's specific activity and, on the other hand, the developed expression to GPX-1 under herceptin treatment, confirms the meaningful induction of apoptosis. Therefore, there would be the title of a drug target in the process of treating this disease.
\end{abstract}

